\title{
Application of infrared thermography in natural convection study
}

\section{by Tomasz S. Wisniewski ${ }^{1}$, Tomasz A. Kowalewski ${ }^{2}$, Marek Rebow ${ }^{1}$}

\author{
${ }^{1}$ Institute of Heat Engineering, Warsaw University of Technology, Nowowiejska 25, 00-665 Warsaw, \\ Poland, e-mail: tswis@itc.pw.edu.pl \\ ${ }^{2}$ Center of Mechanics, IPPT PAN Polish Academy of Sciences, Swietokrzyska 21, 00-049 Warsaw, \\ Poland, e-mail: tkowale@ippt.gov.pl
}

\begin{abstract}
:
The main goal of the study is to test the applicability of Infrared Thermography for verification of Thermal Boundary Conditions at external non-isothermal walls bounding the flow domain. Experimental and numerical studies have been made for transient and steady natural convection in a differentially heated cube filled with water. The analysis is carried out for pure convection of water in the vicinity of the freezing region (cold wall temperature $T_{c}=0^{\circ} \mathrm{C}$ ), and for convection of water accompanied by freezing $\left(T_{c} \leq-10^{\circ} \mathrm{C}\right)$. The opposite hot wall temperature is fixed at $+10^{\circ} \mathrm{C}$.
\end{abstract}

\section{Introduction}

In recent years problems of solid-liquid phase change driven by natural convection and conduction attracted much interest due to their significance for a wide range of technologies and practical applications, like melting and solidification of alloys, crystal growth technology, heat storage or environmental studies of freezing water reservoirs. Several experimental and numerical studies have been dedicated to natural convection in freezing water, indicating the complexity of the problem and necessity of numerical models improvements $[1,2]$. Existing numerical codes appear to fail in modelling details of solidification. The numerical simulations performed recently [3-5] show several differences in the front shape and front pattern.

A possible agent responsible for the discrepancies between calculations and experimental results can be inaccuracy in modelling of the Thermal Boundary Conditions (TBC) at the "passive", non-isothermal side walls. Previous experimental and numerical studies, summarised in [6], indicate that gentle natural convection can be extraordinarily sensitive to small changes in the TBC at the side walls. It appeared that only small variation of the TBCs change the direction and structure of the three-dimensional spiralling motion in the differentially heated cavity. The flow structure could be properly modelled only by applying to the numerical code the temperature fields measured at the side walls.

Complex flow structures associated with phase change problems, their sensitivity to small variation of the flow parameters and initial and boundary conditions, require the application of appropriate experimental methods to validate the numerical results. This in turn requires application of full field acquisition methods, which can give transient data for the velocity and temperature fields of the investigated flow.

The aforementioned discrepancies between numerical and observed results force us to verify Thermal Boundary Conditions imposed on the "passive", non-isothermal side walls of the cavity. To control heat flux through these walls, measurement of both internal and external temperature fields at these walls becomes necessary. Such a possibility is offered by combined application of Infrared and TLCs based Thermography. Temperature fields obtained by IRT at external walls, and internal temperature maps from Particle Image Velocimetry \& Thermometry (PIVT) measurements [7] improve our description of the physical experiment allowing better verification of numerical models. 
To avoid geometrical complications and uncertainty of the thermophysical properties, a simple model of water freezing in a differentially heated cavity is considered. Our main goal is to test the applicability of infrared thermography for verification of the TBCs for the investigated flow. The experimental results obtained are compared with numerical simulation performed using the modified 3-D finite difference code.

\section{Formulation of the problem}

An experimental study has been made of transient and steady convection in a differentially heated cube-shaped enclosure with inner dimension equal to $38 \mathrm{~mm}$ (Fig.1). Distilled water is used as a working fluid. Two opposite vertical walls are assumed isothermal. One of them is held at a temperature $T_{C}=-10^{\circ} \mathrm{C}$. The freezing point of the liquid is $T_{r}=0^{\circ} \mathrm{C}$. In the case of natural convection without phase change the cold wall temperature is set to $T_{c}=0^{\circ} \mathrm{C}$. The opposite vertical wall is held at a temperature $T_{h}=10^{\circ} \mathrm{C}$. The other four walls, made of $6 \mathrm{~mm}$ low thermal conductivity material (Plexiglas) allow the entry of heat from the external air.

To make the heat transfer through the side walls well defined, a constant flux of air generated by a low speed fan was directed at the cavity. The temperature of the air stream is $\mathrm{T}_{\mathrm{ext}}=23.8^{\circ} \mathrm{C}$. Heat transfer from the gas environment through the Plexiglas walls is relatively low and its effect on the internal flow can be considered to be small. However, in the experiment the air temperature is well above the internal fluid temperature, and at least in the near wall regions this may apparently modify the flow.

The initial fluid temperature is equal to $T_{0}=T_{h}=+10^{\circ} \mathrm{C}$. A zero initial velocity flow field is assumed. The convection starts when at time $\mathrm{t}=0$ the cold wall temperature is suddenly decreased to $0^{\circ} \mathrm{C}$ in the case of pure convection or $-10^{\circ} \mathrm{C}$ in the freezing experiments.

\section{Test apparatus and experimental procedure}

The experimental set-up is sketched in Fig. 2. The cubic convection box has two isothermal walls maintained at the prescribed temperatures by anti-freeze coolant flowing through the attached antechambers. The temperature of the cooling and heating liquids are controlled by two circulators. Seeding with thermochromic liquid crystals is used, allowing instantaneous measurement of the velocity and temperature to be made.

For the flow visualization the cavity is illuminated with a $2 \mathrm{~mm}$ thick sheet of white light from a specially constructed halogen lamp. The colour images of TLCs flow seeding are observed in the perpendicular direction by a 3CCD RGB camera. The 24-bit images of $768 \times 564$ pixels resolution are acquired using a 32-bit frame grabber.

The infrared images of the front side wall are acquired with a long wavelength infrared scanner Agema 900, and used to evaluate the temperature field at the one external surface of the wall. The IR Images of the cavity were taken from $110 \mathrm{~mm}$ distance. Due to the space constraints, in the present configuration it is impossible to obtain images from the RGB and the IR camera simultaneously. Hence, a computer controlled system of three stepping motors is used to move the cavity and the RGB camera. It allows the acquisition of flow images of two different vertical cross-sections (near the front wall and at the centre of the cavity), and two infrared images of the front wall. The images are taken fully automatically within 10 seconds. The relatively long relaxation time of the flow allows us to assume that all images are taken at the same instance.

\subsection{Particle Image Velocimetry \& Thermometry}

As mentioned previously Thermochromic Liquid Crystal tracers dispersed in water have been applied to measure both temperature and velocity flow fields. The mean diameter of unencapsulated TLC droplets sphere is about $50 \mu \mathrm{m}$. Their density being close to that of 
water and relative intense light scattering predispose the TLCs suspension as useful tracers for the flow visualization.

TLCs are widely used for surface thermography, however their application for measuring temperature of external walls of the cavity appeared non-practicable. Covering the side walls with a TLC layer or a special TLC sheet would strongly reduce the optical access, and diminish or totally eliminate the possibility of resolving internal temperature and velocity fields. Hence, the Infrared Thermography appears to be the only usable full field measuring technique at the moment.

\subsection{Infrared Thermography}

The system used in our experimental studies consists of a long wavelength (from 8 to 12 $\mu \mathrm{m})$ Agema Thermovision $900 \mathrm{LW}$ infrared scanner. Its spectral response results in low noise at room temperature. Nominal sensitivity of the scanner is equal to $0.08^{\circ} \mathrm{C}$ at $30^{\circ} \mathrm{C}$. Our careful correction procedure, using thermocouples and precise surface temperature measurements allowed us to reach accuracy equal to $0.3^{\circ} \mathrm{C}$.

The scanner image resolution is 200 elements by 136 lines. Each image is digitised in a frame of $272 \times 136$ pixels at 12 bit. Application of software (Erika) allows to select appropriate dynamic range of images and to convert them to 8-bit TIFF images.

The scanner used with a $20^{\circ} \times 10^{\circ}$ lens and a close-up lens has a resolution of $0.23 \mathrm{mrad}$. To improve the spatial resolution of images, the analysed field is acquired in two steps. The first IR image covers the lower half of the investigated side wall, the second image the upper half. The computer controlled system of stepping motors moving the cavity, allows us to minimize the interval between acquisitions of this two images to about $2 \mathrm{~s}$. Specially developed post-processing software is used to merge both half images to one full image of thermal field on the wall with a resulting resolution of $272 \times 272$ pixels.

\section{Numerical Model}

The obtained empirical results are further used to verify the performance and accuracy of the Finite Difference model developed to simulate natural convection with solidification. A modified version of the three-dimensional numerical code FREEZE3D [8] has been used.

When simulating experimental conditions, the main problem which arises is the proper definition of the thermal boundary conditions. The two metal walls are assumed isothermal. The four other walls made of $6 \mathrm{~mm}$ Plexiglas need more complex treatment. To approach as close as possible physical conditions, the code was modified by implementing the side walls into the computational domain. A separate energy equation is solved for the heat transfer from the external air through the four side walls.

Solutions are obtained using $31 \times 31 \times 41$ mesh points. $21^{3}$ mesh points are used for the fluid domain, 5 additional mesh points at each side for the Plexiglas walls, and $21 \times 21 \times 10$ mesh points for the ice domain. To test the mesh dependence selected cases were calculated increasing the number of grid points to $31^{3}$ for the fluid domain.

\section{Results}

\subsection{Natural Convection}

At the beginning, our interest was directed to understanding the transient behaviour of natural convection of water in the vicinity of the freezing point. The experiments start by abruptly dropping the cold wall temperature to $0^{\circ} \mathrm{C}$. The effects of density inversion and of the thermal boundary conditions at non-isothermal walls on the flow structures are studied to compare and eventually improve the numerical code.

A typical flow structure exhibits two circulation regions, where the water density decreases with temperature (upper) and an abnormal density variation (lower). It was found that the 
calculated flow pattern depends strongly on the modelling of the thermal boundary conditions at the side walls. The IR measured distribution of temperature at the external surface of the side wall (Fig. 3) indicates non-uniformity of the heat flux, which could the possible reason for observed discrepancies if adiabatic or constant heat flux model of $T B C$ is used. Only by solving proper, conjugate heat transfer problem for the whole domain including side walls very good agreement with experiments is achieved $[5,6]$.

By combining IR measurements with the liquid crystals thermometry it is possible to obtain additional information on the temperature field on the inner surface of the wall. Hence, resolving temperature differences recorded across the wall, additional hints about the thermophysical properties of the Plexiglas walls as well as about assumed air/wall heat transfer coefficient will be obtained.

\subsection{Freezing of Water}

The pure convection experiments show, as already mentioned, two main circulation regions. The first, driven by normal convection, is located in the upper part of the cavity. It transports the hot liquid up to the top wall and back along the isotherm of the density extreme. The freezing experiments are conducted by starting from the steady natural convection and changing the cold wall temperature from $T_{0}=0^{\circ} \mathrm{C}$ to $T_{0}=-10^{\circ} \mathrm{C}$. When the freezing starts from the developed flow, the fluid/solid interface remain at the isothermal temperature $0^{\circ} \mathrm{C}$. However, interaction of the convective flow with the freezing front causes deformation of initially flat freezing plane (comp. [4]). The hot circulation melts the upper parts of the ice front, reducing the ice growth rate in this region. The abnormal flow circulation, located in the lower right part of the cavity, transports the cold liquid up along the adjacent ice surface and back to the bottom along the isotherm of the density extremum. This cold water circulation only moderately modifies the heat balance at the interface. The convective heat transfer between both upper and lower regions seems to be limited mainly to the upper right corner of the cavity. There, along the colliding cold and warm fluid layers, the heat is transferred from the hot wall to the lower parts of the cavity. The shape of the freezing front reproduces this interaction, almost doubling the ice growth rate at the bottom.

The temperature distribution at the side walls is shown in Fig. 4. Comparing calculated and IR measured fields we may recognise the main features, the characteristic "belly" of the isotherms close to the cold wall. The heat flux through the top and bottom walls increases the temperature in adjacent fluid layers. This in turn modifies the overall circulation pattern, shifting the position of the ice front back to the cold wall.

However, the numerical simulation (Fig. 4) seems to overestimate this effect. Observed and calculated ice fronts are different. Hence, also the temperature and velocity fields are only qualitatively similar. It seems that our numerical efforts to improve modelling of the TBC are not sufficient if the freezing problem is considered. Direct implementation of the IR measured thermal maps of the external surfaces to the code may clear out our doubts about proper modelling of the TBC. However, there are other possible effects responsible for the difficulties encountered in modelling freezing of water, like fluid supercooling, thermal boundary layer at the interfaces and inaccuracy of thermophysical data. Further investigations are necessary for better understanding effects of these factors on the solution of the freezing problem.

\section{Conclusions}

In the study of natural convection of freezing water the infrared technique allowed to measure temperature field on the external surface of non-isothermal wall with an accuracy better then $0.3^{\circ} \mathrm{C}$. It was found that the temperature fields measured at external surfaces of the side walls as well as those of the top/bottom walls are far from being uniform. The observed temperature field of the external surfaces can be combined with the temperature field inside the cavity, visualised with help of liquid crystal tracers. The detailed comparative analysis of 
numerical and experimental results appears to be a unique alternative allowing the understanding of the limitations of the numerical model and giving indications of its improvement.

\section{References}

[1] Fukusako S., Yamada M., Recent advances in research on water-freezing and icemelting problems, Exp. Therm. Fluid Scs., Vol. 6:90-105, 1993

[2] Yeoh GH, Behnia M, de Vahl Davis G, Leonardi E. A numerical study of threedimensional natural convection during freezing of water. Int. J. Num. Meth. Eng., Vol. 30, pp. 899-914, 1990.

[3] Banaszek, J., Rebow, M., Kowalewski T.A. 1997, Fixed grid finite element analysis of solidification, Proc. of Advances in Computational Heat Transfer CHT'97, Edts. G. De Vahl Davis \& E. Leonardi, pp.471-478, Begell House, New York 1998.

[4] Kowalewski TA, Rebow M. Freezing of water in the differentially heated cubic cavity. Int. J. Comp. Fluid Dyn. Vol. 11, pp193-210, 1999.

[5] Giangi M., Stella F., Kowalewski TA. Phase change with convection: fixed grid simulation, Comp. Visual. Scs. Vol.2 (2/3), pp.123-130, 1999.

[6] Kowalewski TA. Experimental validation of numerical codes in thermally driven flows. Adv. in Computational Heat Transfer CHT-97, Edts. G. De Vahl Davis \& E. Leonardi, pp.1-15, Begell House, New York 1998.

[7] Kowalewski TA, Cybulski A., Rebow M. Particle Image Velocimetry and Thermometry in freezing water. 8th Int. Symp. on Flow Visualization, Sorrento, eds. G.M. Carlomagno and I. Grant, CD ROM Proc. ISBN 0953399109, pp.24.1-24.8, Edinburgh, 1998.

[8] Yeoh GH. Natural convection in a solidifying liquid. Ph.D. Thesis, University of New South Wales, 1993.

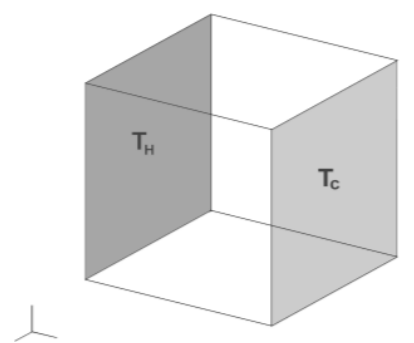

Fig. 1. The cubical box with differentially heated walls

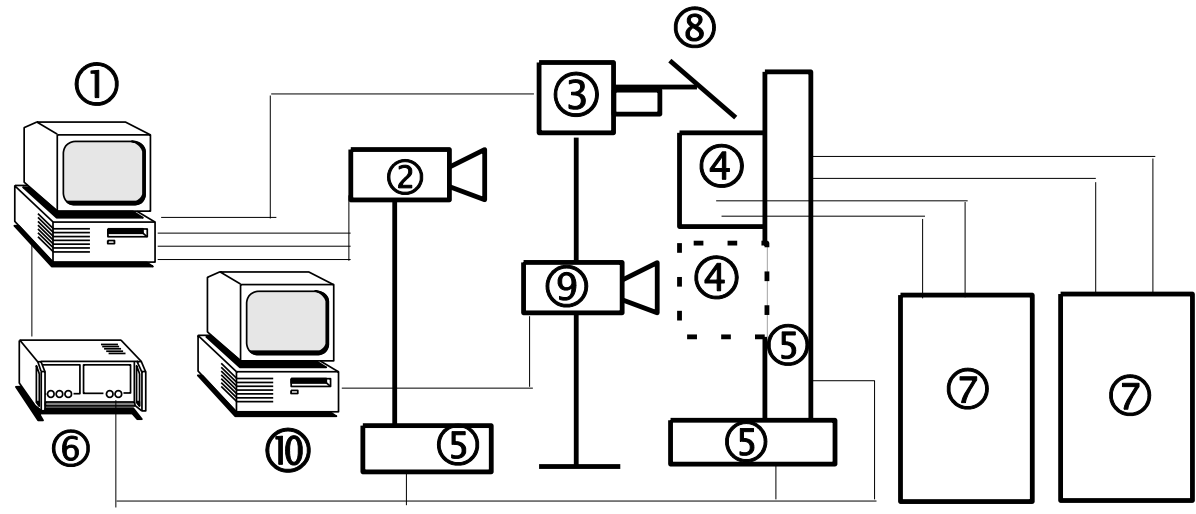

Fig. 2. A schematic view of the experimental set-up. PC (1) with the acquisition card controlling camera (2), halogen lamp (3) and three stepping motors (5) using driver (6). Temperature in the cavity (4) controlled by two circulators (7). Mirror (8). IR camera (9) and IR controller (10) 
http://dx.doi.org/10.21611/qirt.2000.019

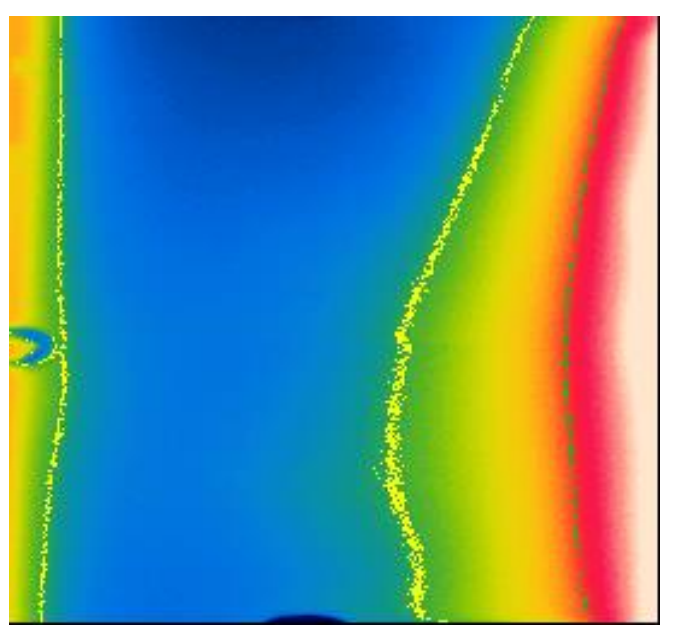

$$
\left.\begin{array}{r}
22.0 \\
18.9 \\
15.7 \\
12.6 \\
9.4 \\
6.3 \\
3.2- \\
0.0
\end{array}\right]
$$

Fig. 3. IR thermographic image of external surface of the side wall with two isothermal lines green $6^{\circ} \mathrm{C}$ and yellow $14^{\circ} \mathrm{C}$ for free convection of water at $2400 \mathrm{~s}$ after start of cooling; Tc $=0^{\circ} \mathrm{C}, \mathrm{Th}=10^{\circ} \mathrm{C}$, Text $=23.8^{\circ} \mathrm{C}$.

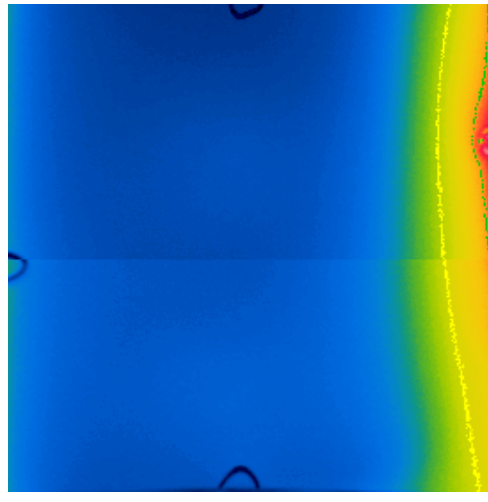

a

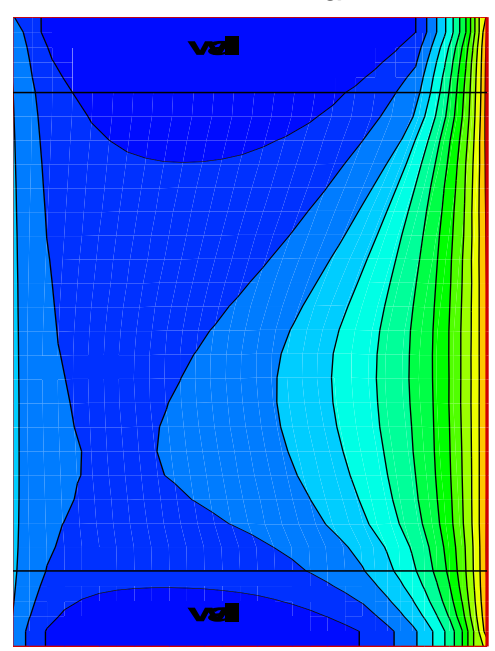

C
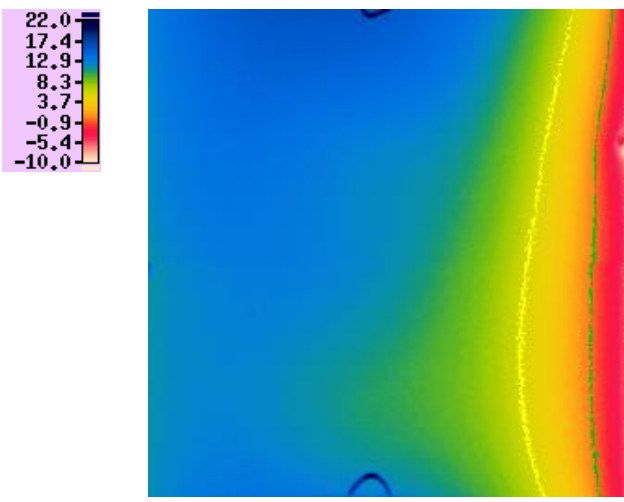

b

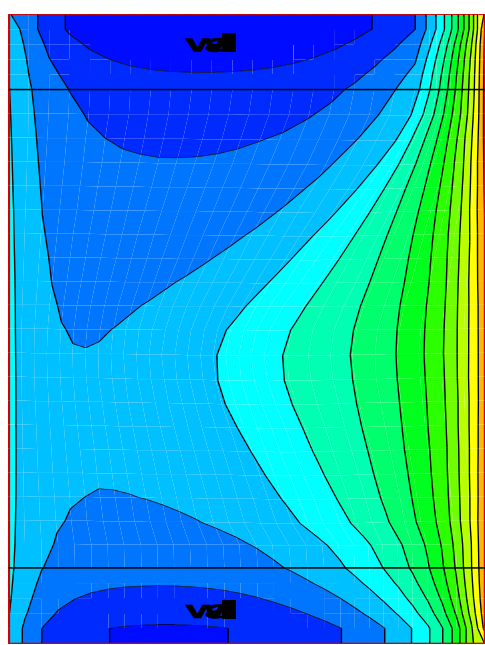

d

Fig. 4. Freezing of water. IR thermographic images with two isothermal lines green $0^{\circ} \mathrm{C}$ and yellow $6{ }^{\circ} \mathrm{C}(\mathrm{a}, \mathrm{b})$ and numerical solution of temperature distribution on the vertical side wall (c, d); time steps 2000s (a, c) and $3600 \mathrm{~s}(\mathrm{~b}, \mathrm{~d})$ after start of cooling; $\mathrm{Tc}=-10^{\circ} \mathrm{C}, \mathrm{Th}=10^{\circ} \mathrm{C}$, Text $=23.8^{\circ} \mathrm{C}$. 